\title{
Determination of adulterants in whey protein food supplements by liquid chromatography coupled to Orbitrap high resolution mass spectrometry
}

\section{Determinação de adulterantes em proteína de soro de leite por cromatografia líquida acoplada à espectrometria de massas de alta resolução do tipo Orbitrap}

\author{
Rafaela Rocha Roiffé ${ }^{1,2}$, Vinicius Figueiredo Sardela², Antônio Luís dos Santos Lima1, \\ Daniely Silva Oliveira², Francisco Radler de Aquino Neto², Keila dos Santos Cople Lima1, \\ Márcia Nogueira da Silva de la Cruz ${ }^{2 *}$ (1)
}

${ }^{1}$ Instituto Militar de Engenharia (IME), Rio de Janeiro/RJ - Brasil

${ }^{2}$ Universidade Federal do Rio de Janeiro (UFRJ), Instituto de Química, Laboratório de Pesquisa, Desenvolvimento e Inovação, Rio de Janeiro/RJ - Brasil

\begin{abstract}
*Corresponding Author: Márcia Nogueira da Silva de la Cruz, Universidade Federal do Rio de Janeiro (UFRJ), Instituto de Química, Departamento de Química Analítica, Laboratório de Pesquisa, Desenvolvimento e Inovação, Avenida Horácio Macedo, 1281, Bloco C, Pólo de Química, Cidade Universitária, Ilha do Fundão, CEP: 21941-598, Rio de Janeiro/RJ - Brasil, e-mail: marcianogueira@iq.ufrj.br
\end{abstract}

Cite as: Roiffé, R. R., Sardela, V. F., Lima, A. L. S., Oliveira, D. S., Aquino Neto, F. R., Lima, K. S. C., \& de la Cruz, M. N. S. (2019). Determination of adulterants in whey protein food supplements by liquid chromatography coupled to Orbitrap high resolution mass spectrometry. Brazilian Journal of Food Technology, 22, e2018206.

https://doi.org/10.1590/1981-6723.20618

\begin{abstract}
Liquid chromatography coupled to Orbitrap high resolution mass spectrometry was shown to be an adequate technique to control the adulteration of whey protein food supplements with prohibited substances, not declared on the labels. An extraction method combined with an instrumental analysis that allowed for the determination of 105 substances in whey protein food supplements, was established. The pre-treatment of the samples consisted of protein precipitation and solid-phase extraction using weak cation exchange functionalized polymeric sorbent cartridges. The samples were directly analyzed by LC-Orbitrap-HRMS. The selectivity, limit of detection, repeatability, recovery, carryover and matrix effect were estimated as the validation parameters. The repeatability obtained was $96.19 \%$ and the recovery $83.80 \%$, but carryover and the matrix effect were not observed. The present method was successfully applied to the analysis of commercial samples, verifying adulteration by diuretics (conivaptan and politiazide) and a stimulant (benfluorex) in seven of the eleven brands evaluated.
\end{abstract}

Keywords: Whey protein food supplement; Adulterants; Pharmacological action; Stimulants; Diuretics; Orbitrap; Method validation. 


\section{Resumo}

A cromatografia líquida acoplada à espectrometria de massas de alta resolução do tipo Orbitrap demonstrou ser uma técnica adequada para o controle de adulteração de substâncias proibidas não declaradas nos rótulos de suplementos proteicos derivados de soro de leite. Foi estabelecido um método de extração combinado a uma análise instrumental que permitiu a determinação de 105 substâncias em suplemento proteico derivado de soro de leite. O pré-tratamento das amostras consistiu na precipitação de proteínas e na extração em fase sólida, utilizando-se cartuchos com sorventes poliméricos baseados em troca catiônica. As amostras foram diretamente analisadas por CL-EMAR-Orbitrap. Foram estimados, como parâmetros de validação, seletividade, limite de detecção, repetitividade, recuperação, arraste e efeito de matriz. A repetitividade obtida foi de $96,19 \%$ e a recuperação foi de $83,80 \%$. Arraste e efeito de matriz não foram observados. O presente método foi aplicado com sucesso na análise de amostras comerciais, nas quais foram verificadas adulterações, em sete das 11 marcas avaliadas, em diuréticos (conivaptan e politiazida) e estimulante (benfluorex).

Palavras-chave: Suplemento alimentar de proteína de soro de leite; Adulterantes; Ação farmacológica; Estimulantes; Diuréticos; Orbitrap; Validação de método.

\section{Introduction}

Over the years, technological and scientific advancement for improving human performance have been studied in different areas (Andrade et al., 2019; Roco \& Bainbridge, 2002; Thomas et al., 2015). However, nutrition is still considered the most relevant aspect regarding muscle building, endurance and strength (Bagchi et al., 2013; McClung \& Murray-Kolb, 2013; Pritchard-Peschek et al., 2013). Due to progress in this area, many athletes, non-athletes and patients with different diseases have been using functional foods to improve their health (Fayh et al., 2013; Horikawa et al., 2013; Mathews, 2018; Maughan et al., 2018; Rondanelli et al., 2016).

The class of food supplements most widely used in the world is that of milk constituents named Whey Protein Food Supplement (WPFS) (Chen et al., 2014; Fayh et al., 2013). The WPFS is obtained from the preparation of cheese, specifically during the casein precipitation step (milk protein) in which it forms a supernatant, the milk serum (Aquino et al., 2017; Chen et al., 2014; Garrido et al., 2016). WPFS shows the following properties: increase in resistance, muscle hypertrophy and decreased body fat (Andrade et al., 2019; Chen et al., 2014; Frestedt et al., 2008; Garrido et al., 2016).

There is no compatible regulation for WPFS between countries (Neves \& Caldas, 2015), and the absence of a specific regulation and better monitoring in the manufacturing process of food supplements, may result in incompatibilities related to the label and content (Andrade et al., 2019; Parra et al., 2011). These mismatches could be related to the quantities of nutrients or other components described on the label or to the presence of substances (intentionally added) that are not reported, resulting in adulteration issues (Andrade et al., 2019; Marcus, 2016).

There are many studies on the adulteration of supplements, and more attention has been focused on adulteration by substances that have pharmacological properties (Garrido et al., 2016; Lu et al., 2010; Martínez-Sanz et al., 2017; Müller et al., 2018; Woo et al., 2013). The main cases of food supplement adulteration are related to the following classes: anabolic agents (provide increases in muscle mass and decreases in body fat); diuretics (decrease body liquids and mask the presence of other substances in the sample); and stimulants (weight loss, increase alertness and reduce fatigue) (Hernandez \& Nahas, 2009; Müller et al., 2018; Neves \& Caldas, 2015; Martínez-Sanz et al., 2017). Martello et al. (2007), described a qualitative liquid chromatography tandem mass spectrometry (LC-MS/MS) method used to detect the following anabolic androgenic steroids (4-androsten-3,17-dion, 4-oestren-3,17-dion, $5 \alpha$-androsten-17 $\beta$-ol-3one, boldenone, nandrolone, nandrolone decanoate, testosterone and testosterone decanoate) and ephedrine 
in food supplements. The LC-MS/MS analysis was carried out using selected reaction monitoring (SRM) in an ion-trap system equipped with an atmospheric pressure chemical ionization (APCI) probe operating in the positive-ion mode. However, this is a target method for a limited number of substances. The method was applied to 64 nutritional supplements and a total of $12.5 \%$ of the nutritional supplements analyzed contained banned substances not declared on the label (anabolic steroids and ephedrine) (Martello et al., 2007). However, some relevant classes of substances such as diuretics and anorectic agents were not evaluated, probably because analysis by SRM only in the positive ionization mode does not allow for the scanning of a comprehensive number of substances. Moreover, for the LC-MS/MS analysis, the liquid-liquid extraction sample preparation using n-pentane and diethyl ether, limited the extraction of acid analytes. In 2010, Lu et al., described a sensitive and specific liquid chromatography-electrospray ionization mass spectrometry (LC/ESI-MS) method for the analysis of 18 drugs used in the treatment of hypertension, including diuretics, as adulterants in dietary supplements (Lu et al., 2010). However, once again it was a very limited procedure regarding the number of substances analyzed.

Multi-target procedures need a more elaborate analytical method, and usually include an ESI interface operating in both positive and negative ionization modes. Moreover, extraction and matrix effects are also a vulnerable point for the routine inspection of WPFS by a single and comprehensive approach. Although, MS/MS experiments allow for the enhancement of sensitivity by applying the selected reaction monitoring (SRM) mode for the determination of selected compounds, the high-resolution mass spectrometry (HRMS) approach enables the specific identification of analytes from the full scan data, making every measurement accessible to subsequent analysis and the search for new, previously not encountered compounds.

Therefore, to determine the presence of adulterants in WPFS, a liquid chromatography method coupled to Orbitrap high resolution mass spectrometry (LC-Orbitrap-HRMS) after solid phase extraction, was optimized and validated to detect the following different classes of substances: anabolic agents, beta-agonists, hormone and metabolic modulators, diuretics and stimulants.

\section{Experimental}

\subsection{Quality assurance}

All analytical and managerial procedures were carried out in an ISO/IEC 17025 standard environment, accredited by the Brazilian National Metrological Institute (BNMI - INMETRO) (Associação Brasileira de Normas Técnicas, 2005).

\subsection{Chemicals and materials}

All solvents used were HPLC grade: methanol, formic acid, ammonium formate and acetic acid (Tedia; Fairfield, USA), and the distilled water was purified by the milli-Q purification system (Millipore, Massachusetts, USA). Reference compounds were purchased mainly from NMI (Sydney, Australia), SigmaAldrich (St. Louis, USA) and Logical (Luckenwalde, Germany) or were kindly donated by other anti-doping laboratories. The Strata-X-CW, weak cation mixed mode polymeric sorbent (30 mg, $3 \mathrm{~mL}$ ) SPE cartridges (São Paulo, Brazil) were purchased from Phenomenex. The internal standards mefruside, methyltestosterone, n-methylhexanamine, buspirone hydrochloride, 4-methylefedrine-D3 $\mathrm{HCl}$, and 7-propyltheophylline were purchased from Sigma-Aldrich (St. Louis, USA). 


\subsection{Standard solutions}

The fortification solution containing the reference standards consisted of a mixture of the following classes of substances: anabolic agents, beta-agonists, hormone and metabolic modulators, diuretics and masking

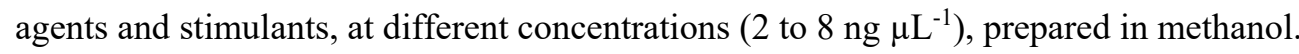

The internal standard (IS) solution consisted of a mixture of the substances mefruside, methyltestosterone, n-methylhexanamine, propyl-theophylline and 7-propyl-theophylline at different concentrations (6 to $20 \mathrm{ng} \mu \mathrm{L}^{-1}$ ), all dissolved in methanol.

\subsection{Sample preparation}

Eleven brands of WPFS were purchased from local Brazilian markets and $20 \mathrm{mg}$ of each sample dissolved in $2 \mathrm{~mL}$ of water. Three controls were prepared: reagent blank (water), negative control (matrix without the analytes) and positive control (matrix spiked with $50 \mu \mathrm{L}$ of fortification solution). The samples and controls were homogenized during $20 \mathrm{~s}$ and centrifuged for $20 \mathrm{~min}$ at $1.5 \mathrm{x}$ G. One aliquot of the $10 \mu \mathrm{L}$ in each test tube was transferred to a conical Eppendorf tube and $50 \mu \mathrm{L}$ of the $2 \%(\mathrm{v} / \mathrm{v})$ acetic acid solution added. The tubes were centrifuged for $5 \mathrm{~min}$ at $1.5 \mathrm{x} \mathrm{G}$ and stored at $2{ }^{\circ} \mathrm{C}$ to $6{ }^{\circ} \mathrm{C}$ for the subsequent reconstitution of the extract. The second aliquot taken from the supernatant obtained from each initial sample was transferred to a new test tube, $20 \mu \mathrm{L}$ of the internal standard solution added and the contents homogenized by vortex for $20 \mathrm{~s}$. In sequence, the solid-phase extraction (SPE) step was carried out. The SPE cartridges were conditioned with $1 \mathrm{~mL}$ of methanol and $1 \mathrm{~mL}$ of Milli-Q water; the sample was applied and the cartridges washed with $1 \mathrm{~mL}$ of Milli-Q water and $1 \mathrm{~mL}$ of $50 \%(\mathrm{v} / \mathrm{v})$ methanol in water. The analytes were eluted with $1 \mathrm{~mL}$ of $5 \%(\mathrm{v} / \mathrm{v})$ formic acid in methanol. All samples were evaporated under a nitrogen flow at $45{ }^{\circ} \mathrm{C}$. The first aliquot was added to the dried residue and the mixture homogenized by vortex for $20 \mathrm{~s}$, transferred to vials with the inserts and refrigerated at $4{ }^{\circ} \mathrm{C}$ for $4 \mathrm{~h}$. The supernatants were transferred to new vials with inserts and injected into the chromatographic system.

\subsection{Instrumentation}

The liquid chromatography system was an Accela LC liquid chromatography (Thermo Scientific, Bremen, Germany), with an Accela 1250 pump and auto sampler fixed at $10{ }^{\circ} \mathrm{C}$. The column was a Zorbax SB-C 18 one, $3.0 \mathrm{~mm} \times 50 \mathrm{~mm}, 1.8 \mu \mathrm{m}$ (Agilent, Böblingen, Germany). The mobile phases were $0.1 \%$ ammonium formate $/ 0.1 \%$ formic acid in water (A) and $0.1 \%$ formic acid in methanol (B). The gradient program was as follows: $95 \%$ A for $0.5 \mathrm{~min}$, then decreasing linearly to $90 \%$ at $10.0 \mathrm{~min}$, then to $0 \%$ at $11.1 \mathrm{~min}$, followed by an increase to the initial concentration of $95 \% \mathrm{~A}$ at $14.0 \mathrm{~min}$. The total run time was thus $14 \mathrm{~min}$. The column was maintained at $40{ }^{\circ} \mathrm{C}$, the flow rate constant at $600 \mu \mathrm{L} \mathrm{min}^{-1}$ and the injection volume was $0.5 \mu \mathrm{L}$.

The LC effluent was pumped to a Q Exactive Orbitrap-based high resolution mass spectrometer (Thermo Scientific, Bremen Germany) operating in the positive-negative polarity switching mode and equipped with an electrospray ionization (ESI) source. The nitrogen gas flow and auxiliary gas were set to 60 and 20 (arbitrary units), respectively. The capillary temperature was $380{ }^{\circ} \mathrm{C}$, the spray voltage $3900 \mathrm{kV}-2900 \mathrm{kV}$ and the capillary voltage $3.9 \mathrm{~V}$ or $-2.9 \mathrm{~V}$, in the positive or negative modes, respectively. The instrument was operated in the full scan mode from $\mathrm{m} / \mathrm{z} 100$ to 620 and from 70 to 630 , in the positive and negative modes, respectively, at 70,000 resolution power. The automatic gain control (AGC) was $10^{6}$. The performance of the Orbitrap in both the positive and negative ionization modes was evaluated daily.

The data obtained after the LC-Orbitrap-HRMS analyses were processed using the Qual Browser (Thermo Electron, San Jose, CA), applying a 5 ppm tolerance error and FULL-MS acquisition. In addition, the formula calculator used included carbon, hydrogen and oxygen atoms to provide chemical formula and saturation 
values (ring double-bonds equivalent - RDBE) for the precursor ions $[\mathrm{M}+\mathrm{H}]^{+}$and $[\mathrm{M}+\mathrm{H}]^{-}$. A comparison between the theoretical and experimental precursor molecular mass values was evaluated in the identification of molecule structures. The instrumental conditions used were established according to anti-doping control (Sardela et al., 2018).

\subsection{Method validation}

The method was validated by qualitative screening, according to the internal validation protocol of the Laboratório Brasileiro de Controle de Dopagem, based on the DOC-CGCRE 008 protocol of the Instituto Nacional de Metrologia, Qualidade e Tecnologia. The selectivity evaluated ten different WPFS samples, declared as negative, and verified the absence of interfering substances in the retention times of each analyte. The limit of detection was evaluated by preparing 10 different samples spiked with $10 \%$ of the usual concentration and 10 different samples spiked with $50 \%$ of the usual concentration. The repeatability was determined using 7 replicates of WPFS, and the peak area values were expressed according to the relative standard deviation of each substance monitored. The recovery was evaluated by preparing 7 replicates spiked before the solid phase extraction step (repeatability) and 7 replicates spiked after the solid phase extraction step. The carryover was verified by an analysis of the spiked WPFS sample with twice the fortification solution concentration between 2 blank WPFS samples. The matrix effect evaluated 10 different WPFS samples, declared negative, which were spiked with the monitored analytes.

\subsection{Application to real samples}

The optimized and validated method by LC-HRMS was applied to the analysis of eleven commercial samples of WPFS products.

\section{Results and discussion}

\subsection{Sample preparation}

The WPFS samples contain a high concentration of the proteins $\beta$-lactoglobulin, $\alpha$-lactalbumin, serum albumin, immunoglobulins and glycomacropeptides, as well as other minor proteins such as lactoperoxidase, lactoferrin, $\beta$-microglobulin, lysozyme, insulin-like growth factor and others (Haraguchi et al., 2006). These proteins should be removed before injecting the sample into the chromatographic system. The presence of those compounds may clog the SPE column or the analytical column, due to precipitation, reducing the life of the column and competing for the electrospray ionization source, thus interfering in substance detection. Hence, it is indispensable to carry out sample pre-treatment procedures to remove these proteins, without loss of the target analytes. Thus a solvent precipitation step before the solid phase extraction was tested with different solvents, in order to reduce the amount of protein in the matrix, but not remove the target substances. Cold water, acetonitrile and a combination of acetonitrile/water $(50 \%-\mathrm{v} / \mathrm{v})$ were tested and the final recoveries of the substances compared based on their peak areas.

Acetonitrile and acetonitrile/water were not effective, because they also removed some target substances, especially the diuretics. Only the use of the cold water dissolved the WPFS and, at the same time, eliminated the excess of proteins and allowed for the detection of all the target substances with good recovery.

\subsection{Method validation}

Selectivity verifies the absence of interference in the retention times of the monitored substances. No significant interference in the retention times was observed for the target substances. 
Table 1. Chemical formula, polarity, retention times ( $\left.\mathrm{t}_{\mathrm{R}}\right)$, theoretical masses $(\mathrm{m} / \mathrm{z})$, sample concentration (SC), repeatability, recovery, limit of detection (LOD) and matrix effect of the monitored compounds.

\begin{tabular}{|c|c|c|c|c|c|c|c|c|c|}
\hline & & & & & SC & Repeatability & Extraction & LOD & Matrix \\
\hline Compound & Chemical Formula & Polarity & $t_{R}(\min )$ & $m / z$ & $\left(\mathrm{ng} \mathrm{g}^{-1}\right)$ & $(\%)$ & $\begin{array}{l}\text { yield } \\
(\%)\end{array}$ & $\left(\right.$ ng. $\left.^{-1}\right)$ & $\begin{array}{c}\text { interference } \\
(\%)\end{array}$ \\
\hline Andarine & $\mathrm{C}_{19} \mathrm{H}_{18} \mathrm{~F}_{3} \mathrm{~N}_{3} \mathrm{O}_{6}$ & - & 6.07 & 440.10749 & 12.5 & 14.34 & 99.0 & 1.25 & 0.30 \\
\hline Gestrinone & $\mathrm{C}_{21} \mathrm{H}_{24} \mathrm{O}_{2}$ & + & 7.00 & 309.18491 & 12.5 & 18.00 & 96.8 & 1.25 & 0.15 \\
\hline Methyldienolone & $\mathrm{C}_{19} \mathrm{H}_{26} \mathrm{O}_{2}$ & + & 6.96 & 287.20056 & 12.5 & 10.06 & 95.8 & 1.25 & 0.11 \\
\hline Methyltrienolone & $\mathrm{C}_{19} \mathrm{H}_{24} \mathrm{O}_{2}$ & + & 6.90 & 285.18491 & 12.5 & 6.87 & 95.1 & 1.25 & 0.14 \\
\hline Ostarine & $\mathrm{C}_{19} \mathrm{H}_{14} \mathrm{~F}_{3} \mathrm{~N}_{3} \mathrm{O}_{3}$ & - & 6.46 & 388.09145 & 12.5 & 21.58 & 96.2 & 1.25 & 0.12 \\
\hline Oxandrolone & $\mathrm{C}_{19} \mathrm{H}_{30} \mathrm{O}_{3}$ & + & 6.60 & 307.22677 & 12.5 & 8.26 & 95.7 & 1.25 & 0.13 \\
\hline Tetrahydrogestrinone & $\mathrm{C}_{21} \mathrm{H}_{28} \mathrm{O}_{2}$ & + & 7.87 & 313.21621 & 12.5 & 18.49 & 96.3 & 1.25 & 0.14 \\
\hline Bamethan & $\mathrm{C}_{12} \mathrm{H}_{19} \mathrm{NO}_{2}$ & + & 1.80 & 210.14886 & 50.0 & 1.94 & 91.6 & 5.00 & 1.17 \\
\hline Formoterol & $\mathrm{C}_{19} \mathrm{H}_{24} \mathrm{~N}_{2} \mathrm{O}_{4}$ & + & 3.29 & 345.18088 & 100.0 & 5.38 & 92.0 & 10.00 & 1.12 \\
\hline Isoxsuprine & $\mathrm{C}_{18} \mathrm{H}_{23} \mathrm{NO}_{3}$ & + & 3.71 & 302.17507 & 50.0 & 5.69 & 94.1 & 5.00 & 0.90 \\
\hline Metaproterenol & $\mathrm{C}_{11} \mathrm{H}_{17} \mathrm{NO}_{3}$ & + & 0.49 & 212.12812 & 50.0 & 15.86 & 96.7 & 5.00 & 2.63 \\
\hline Procaterol & $\mathrm{C}_{16} \mathrm{H}_{22} \mathrm{~N}_{2} \mathrm{O}_{3}$ & + & 1.96 & 291.17032 & 50.0 & 8.86 & 95.2 & 5.00 & 1.17 \\
\hline Ritodrine & $\mathrm{C}_{17} \mathrm{H}_{21} \mathrm{NO}_{3}$ & + & 2.02 & 288.15942 & 50.0 & 5.53 & 97.8 & 5.00 & 1.24 \\
\hline Salmeterol & $\mathrm{C}_{25} \mathrm{H}_{37} \mathrm{NO}_{4}$ & + & 6.33 & 416.27954 & 50.0 & 10.16 & 90.0 & 5.00 & 0.12 \\
\hline Aminoglutethimide & $\mathrm{C}_{10} \mathrm{H}_{12} \mathrm{~N}$ & + & 2.31 & 146.09642 & 50.0 & 10.80 & 92.8 & 5.00 & 0.94 \\
\hline Anastrazole & $\mathrm{C}_{17} \mathrm{H}_{19} \mathrm{~N}_{5}$ & + & 4.80 & 294.17132 & 50.0 & 7.44 & 92.8 & 5.00 & 0.14 \\
\hline Androstatrienedione & $\mathrm{C}_{19} \mathrm{H}_{24} \mathrm{O}_{2}$ & + & 6.20 & 283.16926 & 50.0 & 9.49 & 95.7 & 5.00 & 0.13 \\
\hline Exemestane & $\mathrm{C}_{20} \mathrm{H}_{24} \mathrm{O}_{2}$ & + & 6.77 & 297.18490 & 50.0 & 13.45 & 98.5 & 5.00 & 0.12 \\
\hline Flutamide & $\mathrm{C}_{11} \mathrm{H}_{11} \mathrm{~F}_{3} \mathrm{~N}_{2} \mathrm{O}_{3}$ & - & 6.39 & 275.06381 & 50.0 & 13.42 & 97.4 & 5.00 & 0.29 \\
\hline Fulvestrant & $\mathrm{C}_{32} \mathrm{H}_{47} \mathrm{~F}_{5} \mathrm{O}_{3} \mathrm{~S}$ & + & 8.79 & 607.32388 & 50.0 & 32.86 & 75.4 & 5.00 & 0.08 \\
\hline Gw501516 & $\mathrm{C}_{21} \mathrm{H}_{18} \mathrm{~F}_{3} \mathrm{NO}_{3} \mathrm{~S}_{2}$ & + & 8.70 & 454.07530 & 50.0 & 37.94 & 75.3 & 5.00 & 0.10 \\
\hline Raloxifene & $\mathrm{C}_{28} \mathrm{H}_{27} \mathrm{NO}_{4} \mathrm{~S}$ & + & 5.00 & 474.17336 & 50.0 & 17.68 & 89.6 & 5.00 & 0.43 \\
\hline Bendroflumethiazide & $\mathrm{C}_{15} \mathrm{H}_{14} \mathrm{~F}_{3} \mathrm{~N}_{3} \mathrm{O}_{4} \mathrm{~S}_{2}$ & - & 4.85 & 420.03051 & 125.0 & 10.21 & 92.7 & 12.50 & 0.26 \\
\hline Benzbromarone & $\mathrm{C}_{17} \mathrm{H}_{12} \mathrm{Br}_{2} \mathrm{O}_{3}$ & + & 8.48 & 422.92260 & 125.0 & 25.83 & 77.4 & 62.50 & 0.14 \\
\hline Benzthiazide & $\mathrm{C}_{15} \mathrm{H}_{14} \mathrm{CIN}_{3} \mathrm{O}_{4} \mathrm{~S}_{3}$ & - & 4.75 & 429.97622 & 62.5 & 10.73 & 94.9 & 6.25 & 0.11 \\
\hline Bumetanide & $\mathrm{C}_{17} \mathrm{H}_{20} \mathrm{~N}_{2} \mathrm{O}_{5} \mathrm{~S}$ & - & 6.26 & 363.10202 & 125.0 & 34.93 & 29.5 & 62.50 & 0.13 \\
\hline Chlorothiazide & $\mathrm{C}_{7} \mathrm{H}_{8} \mathrm{CIN}_{3} \mathrm{O}_{4} \mathrm{~S}_{2}$ & - & 0.95 & 293.94155 & 125.0 & 22.76 & 5.6 & 12.50 & 1.21 \\
\hline Chlorthalidone & $\mathrm{C}_{14} \mathrm{H}_{11} \mathrm{CIN}_{2} \mathrm{O}_{4} \mathrm{~S}$ & - & 3.61 & 337.00553 & 125.0 & 13.94 & 9.8 & 62.50 & 0.34 \\
\hline
\end{tabular}


Determination of adulterants in whey protein food supplements by liquid chromatography coupled to Orbitrap high resolution mass spectrometry Roiffé, R. R. et al.

Table 1. Continued...

\begin{tabular}{|c|c|c|c|c|c|c|c|c|c|}
\hline & & & & & $\mathrm{SC}$ & Repeatability & Extraction & LOD & Matrix \\
\hline Compound & Chemical Formula & Polarity & $t_{R}(\min )$ & $m / z$ & $\left(\mathrm{ng} \mathrm{g}^{-1}\right)$ & $(\%)$ & $\begin{array}{l}\text { yield } \\
(\%)\end{array}$ & $\left(\right.$ ng..$\left.^{-1}\right)$ & $\begin{array}{c}\text { interference } \\
(\%)\end{array}$ \\
\hline Clopamide & $\mathrm{C}_{14} \mathrm{H}_{20} \mathrm{ClN}_{3} \mathrm{O}_{3} \mathrm{~S}$ & - & 4.10 & 344.08411 & 62.5 & 12.04 & 40.8 & 6.25 & 0.10 \\
\hline Conivaptan & $\mathrm{C}_{32} \mathrm{H}_{26} \mathrm{~N}_{4} \mathrm{O}_{2}$ & + & 5.84 & 499.21285 & 125.0 & 12.49 & 88.7 & 12.50 & 0.21 \\
\hline Cyclopenthiazide & $\mathrm{C}_{13} \mathrm{H}_{18} \mathrm{ClN}_{3} \mathrm{O}_{4} \mathrm{~S}_{2}$ & - & 5.30 & 378.03545 & 125.0 & 11.98 & 97.1 & 12.50 & 0.13 \\
\hline Cyclothiazide & $\mathrm{C}_{4} \mathrm{H}_{16} \mathrm{ClN}_{3} \mathrm{O}_{4} \mathrm{~S}_{2}$ & - & 4.88 & 388.01979 & 125.0 & 11.34 & 95.4 & 12.50 & 0.25 \\
\hline Diclofenamide & $\mathrm{C}_{6} \mathrm{H}_{6} \mathrm{Cl}_{2} \mathrm{~N}_{2} \mathrm{O}_{4} \mathrm{~S}_{2}$ & - & 2.52 & 302.90733 & 125.0 & 16.94 & 6.8 & 12.50 & 0.51 \\
\hline Etacrynic acid & $\mathrm{C}_{13} \mathrm{H}_{12} \mathrm{Cl}_{2} \mathrm{O}_{4}$ & - & 6.47 & 301.00399 & 125.0 & 35.81 & 15.3 & 12.50 & 0.38 \\
\hline Hydrochlorothiazide & $\mathrm{C}_{7} \mathrm{H}_{8} \mathrm{CIN}_{3} \mathrm{O}_{4} \mathrm{~S}_{2}$ & - & 1.18 & 295.95720 & 250.0 & 26.58 & 5.3 & 25.00 & 0.00 \\
\hline Hydroflumethiazide & $\mathrm{C}_{8} \mathrm{H}_{8} \mathrm{~F}_{3} \mathrm{~N}_{3} \mathrm{O}_{4} \mathrm{~S}_{2}$ & - & 1.75 & 329.98356 & 62.5 & 24.89 & 4.1 & 6.25 & 0.24 \\
\hline Lixivaptan & $\mathrm{C}_{27} \mathrm{H}_{21} \mathrm{CIFN}_{3} \mathrm{O}_{2}$ & + & 7.52 & 474.13791 & 125.0 & 38.07 & 81.4 & 62.50 & 0.11 \\
\hline Methazolamide & $\mathrm{C}_{5} \mathrm{H}_{8} \mathrm{~N}_{4} \mathrm{O}_{3} \mathrm{~S}_{2}$ & - & 2.12 & 234.99650 & 125.0 & 31.49 & 4.4 & 12.50 & 0.24 \\
\hline Methyclothiazide & $\mathrm{C}_{9} \mathrm{H}_{11} \mathrm{Cl}_{2} \mathrm{~N}_{3} \mathrm{O}_{4} \mathrm{~S}_{2}$ & + & 3.37 & 359.96408 & 500.0 & 5.36 & 117.7 & 250.00 & 0.38 \\
\hline Piretanide & $\mathrm{C}_{17} \mathrm{H}_{18} \mathrm{~N}_{2} \mathrm{O}_{5} \mathrm{~S}$ & - & 5.84 & 361.08637 & 125.0 & 30.09 & 12.4 & 62.50 & 0.31 \\
\hline Polythiazide & $\mathrm{C}_{11} \mathrm{H}_{13} \mathrm{ClF}_{3} \mathrm{~N}_{3} \mathrm{O}_{4} \mathrm{~S}_{3}$ & - & 4.81 & 437.96360 & 62.5 & 8.62 & 95.9 & 6.25 & 0.14 \\
\hline Probenecid & $\mathrm{C}_{13} \mathrm{H}_{19} \mathrm{NO}_{4} \mathrm{~S}$ & - & 6.24 & 284.09620 & 62.5 & 23.93 & 27.1 & 6.25 & 0.13 \\
\hline Spironolactone & $\mathrm{C}_{24} \mathrm{H}_{32} \mathrm{O}_{4} \mathrm{~S}$ & + & 6.80 & 341.21112 & 62.5 & 13.94 & 99.3 & 6.25 & 0.17 \\
\hline Torasemide & $\mathrm{C}_{16} \mathrm{H}_{20} \mathrm{~N}_{4} \mathrm{O}_{3} \mathrm{~S}$ & - & 4.77 & 347.11833 & 62.5 & 8.25 & 89.4 & 6.25 & 0.23 \\
\hline Triamterene & $\mathrm{C}_{12} \mathrm{H}_{11} \mathrm{~N}_{7}$ & + & 2.96 & 254.11487 & 62.5 & 5.24 & 94.2 & 6.25 & 1.49 \\
\hline Trichlormethiazide & $\mathrm{C}_{8} \mathrm{H}_{8} \mathrm{Cl}_{3} \mathrm{~N}_{3} \mathrm{O}_{4} \mathrm{~S}_{2}$ & - & 3.06 & 377.89490 & 125.0 & 16.54 & 21.5 & 12.50 & 0.44 \\
\hline Xipamide & $\mathrm{C}_{15} \mathrm{H}_{15} \mathrm{CIN}_{2} \mathrm{O}_{4} \mathrm{~S}$ & - & 5.61 & 353.03683 & 62.5 & 19.16 & 24.6 & 6.25 & 0.15 \\
\hline (s)-2-aminooctane & $\mathrm{C}_{8} \mathrm{H}_{19} \mathrm{~N}$ & + & 3.90 & 130.15902 & 125.0 & 2.94 & 91.5 & 12.50 & 1.19 \\
\hline 3,3-diphenylpropylamine & $\mathrm{C}_{15} \mathrm{H}_{17} \mathrm{~N}$ & + & 4.68 & 212.14338 & 125.0 & 3.53 & 90.1 & 12.50 & 0.69 \\
\hline 4-fluoroamphetamine & $\mathrm{C}_{9} \mathrm{H}_{12} \mathrm{FN}$ & + & 2.00 & 154.10265 & 125.0 & 4.50 & 89.1 & 12.50 & 1.10 \\
\hline Amiphenazole & $\mathrm{C}_{9} \mathrm{H}_{9} \mathrm{~N}_{3} \mathrm{~S}$ & + & 1.59 & 192.05899 & 125.0 & 14.42 & 101.8 & 62.50 & 1.37 \\
\hline Benfluorex & $\mathrm{C}_{19} \mathrm{H}_{20} \mathrm{~F}_{3} \mathrm{NO}_{2}$ & + & 5.80 & 352.15189 & 125.0 & 9.96 & 86.5 & 12.50 & 0.12 \\
\hline Benzphetamine & $\mathrm{C}_{17} \mathrm{H}_{21} \mathrm{~N}$ & + & 4.18 & 240.17468 & 125.0 & 4.24 & 91.2 & 12.50 & 0.83 \\
\hline Benzylpiperazine & $\mathrm{C}_{11} \mathrm{H}_{16} \mathrm{~N}_{2}$ & + & 0.90 & 177.13863 & 31.25 & 11.67 & 87.9 & 3.16 & 5.66 \\
\hline Carphedon & $\mathrm{C}_{12} \mathrm{H}_{14} \mathrm{~N}_{2} \mathrm{O}_{2}$ & + & 3.18 & 219.11280 & 125.0 & 12.71 & 36.1 & 12.50 & 0.13 \\
\hline Cathine & $\mathrm{C}_{9} \mathrm{H}_{13} \mathrm{NO}$ & + & 1.36 & 134.09640 & 250.0 & 5.69 & 88.3 & 25.00 & 1.70 \\
\hline Chlorphentermine & $\mathrm{C}_{10} \mathrm{H}_{14} \mathrm{ClN}$ & + & 3.62 & 184.08875 & 125.0 & 3.76 & 91.3 & 12.50 & 1.45 \\
\hline Clobenxorex & $\mathrm{C}_{16} \mathrm{H}_{18} \mathrm{ClN}$ & + & 4.93 & 260.12005 & 62.5 & 4.55 & 92.1 & 6.25 & 0.57 \\
\hline Cocaine & $\mathrm{C}_{17} \mathrm{H}_{21} \mathrm{NO}_{4}$ & + & 3.24 & 304.15433 & 125.0 & 4.45 & 96.2 & 12.50 & 1.26 \\
\hline
\end{tabular}


Determination of adulterants in whey protein food supplements by liquid chromatography coupled to Orbitrap high resolution mass spectrometry Roiffé, R. R. et al.

Table 1. Continued...

\begin{tabular}{|c|c|c|c|c|c|c|c|c|c|}
\hline & & & & & SC & Repeatability & Extraction & LOD & Matrix \\
\hline Compound & Chemical Formula & Polarity & $t_{R}(\min )$ & $m / z$ & $\left(\mathrm{ng} \mathrm{g}^{-1}\right)$ & (\%) & $\begin{array}{l}\text { yield } \\
(\%)\end{array}$ & $\left(\right.$ ng..$\left.^{-1}\right)$ & $\begin{array}{c}\text { interference } \\
(\%)\end{array}$ \\
\hline Cropropamide & $\mathrm{C}_{13} \mathrm{H}_{24} \mathrm{~N}_{2} \mathrm{O}_{2}$ & + & 5.10 & 241.19105 & 125.0 & 5.10 & 78.0 & 12.50 & 0.13 \\
\hline Crotethamide & $\mathrm{C}_{12} \mathrm{H}_{22} \mathrm{~N}_{2} \mathrm{O}_{2}$ & + & 4.25 & 227.17540 & 125.0 & 15.34 & 70.6 & 12.50 & 2.00 \\
\hline Cyclazodone & $\mathrm{C}_{12} \mathrm{H}_{12} \mathrm{~N}_{2} \mathrm{O}_{2}$ & + & 4.04 & 217.09715 & 125.0 & 7.37 & 87.3 & 12.50 & 0.25 \\
\hline Dobutamine & $\mathrm{C}_{18} \mathrm{H}_{23} \mathrm{NO}_{3}$ & + & 2.73 & 302.17507 & 125.0 & 7.38 & 95.6 & 12.50 & 1.56 \\
\hline Etamivan & $\mathrm{C}_{12} \mathrm{H}_{17} \mathrm{NO}_{3}$ & + & 4.16 & 224.12812 & 62.5 & 11.03 & 52.6 & 6.25 & 0.20 \\
\hline Etilefrine & $\mathrm{C}_{10} \mathrm{H}_{15} \mathrm{NO}_{2}$ & + & 0.55 & 182.11756 & 125.0 & 7.64 & 96.6 & 12.50 & 3.12 \\
\hline Famprofazone & $\mathrm{C}_{24} \mathrm{H}_{31} \mathrm{~N}_{3} \mathrm{O}$ & + & 6.40 & 378.25399 & 62.5 & 6.85 & 88.5 & 6.25 & 0.28 \\
\hline Fenbrutazate & $\mathrm{C}_{23} \mathrm{H}_{29} \mathrm{NO}_{3}$ & + & 6.30 & 368.22202 & 62.5 & 8.59 & 87.8 & 6.25 & 0.24 \\
\hline Fencamine & $\mathrm{C}_{20} \mathrm{H}_{28} \mathrm{~N}_{6} \mathrm{O}_{2}$ & + & 3.26 & 385.23465 & 62.5 & 7.79 & 92.9 & 6.25 & 0.89 \\
\hline Fenethyline & $\mathrm{C}_{18} \mathrm{H}_{23} \mathrm{~N}_{5} \mathrm{O}_{2}$ & + & 3.53 & 342.19245 & 125.0 & 3.43 & 95.3 & 12.50 & 0.82 \\
\hline Fenfluramine & $\mathrm{C}_{12} \mathrm{H}_{16} \mathrm{~F}_{3} \mathrm{~N}$ & + & 3.93 & 232.13076 & 125.0 & 3.25 & 90.4 & 12.50 & 1.19 \\
\hline Fenproporex & $\mathrm{C}_{12} \mathrm{H}_{16} \mathrm{~N}_{2}$ & + & 1.92 & 189.13863 & 62.5 & 1.72 & 89.8 & 6.25 & 1.13 \\
\hline Flephedrone & $\mathrm{C}_{10} \mathrm{H}_{12} \mathrm{FNO}$ & + & 4.33 & 182.09757 & 125.0 & 23.19 & 108.7 & 12.50 & 1.58 \\
\hline Furfenorex & $\mathrm{C}_{15} \mathrm{H}_{19} \mathrm{NO}$ & + & 3.46 & 230.15394 & 125.0 & 4.24 & 88.8 & 12.50 & 1.17 \\
\hline Heptaminol & $\mathrm{C}_{8} \mathrm{H}_{19} \mathrm{NO}$ & + & 1.30 & 146.15394 & 250.0 & 6.18 & 87.6 & 25.00 & 2.00 \\
\hline Isometheptene & $\mathrm{C}_{24} \mathrm{H}_{48} \mathrm{~N}_{2} \mathrm{O}_{8}$ & + & 3.02 & 142.15903 & 125.0 & 2.95 & 90.4 & 12.50 & 1.55 \\
\hline Mefenorex & $\mathrm{C}_{12} \mathrm{H}_{18} \mathrm{CIN}$ & + & 3.37 & 212.12005 & 62.5 & 2.62 & 90.3 & 6.25 & 1.40 \\
\hline Mephedrone & $\mathrm{C}_{11} \mathrm{H}_{15} \mathrm{NO}$ & + & 2.50 & 178.12264 & 125.0 & 3.24 & 92.9 & 12.50 & 1.46 \\
\hline Mesocarb & $\mathrm{C}_{18} \mathrm{H}_{18} \mathrm{~N}_{4} \mathrm{O}_{2}$ & + & 6.20 & 323.15025 & 125.0 & 14.89 & 93.0 & 12.50 & 0.13 \\
\hline Methoxyphenamine & $\mathrm{C}_{11} \mathrm{H}_{17} \mathrm{NO}$ & + & 2.77 & 180.13829 & 62.5 & 3.15 & 92.6 & 6.25 & 1.48 \\
\hline Methylenodioxyamfetamine & $\mathrm{C}_{10} \mathrm{H}_{13} \mathrm{NO}_{2}$ & + & 2.09 & 163.07540 & 125.0 & 5.92 & 38.8 & 12.50 & 1.23 \\
\hline Methylenodioxymethamfetamine & $\mathrm{C}_{11} \mathrm{H}_{15} \mathrm{NO}_{2}$ & + & 2.14 & 194.11756 & 62.5 & 4.69 & 91.1 & 6.25 & 1.62 \\
\hline Methylenodioxy-n-ethylamfetamine & $\mathrm{C}_{12} \mathrm{H}_{17} \mathrm{NO}_{2}$ & + & 2.46 & 208.13321 & 125.0 & 2.87 & 92.9 & 12.50 & 1.47 \\
\hline Methylephedrine & $\mathrm{C}_{11} \mathrm{H}_{17} \mathrm{NO}$ & + & 1.61 & 180.13829 & 125.0 & 3.68 & 94.4 & 12.50 & 1.30 \\
\hline Methylphenidate & $\mathrm{C}_{14} \mathrm{H}_{19} \mathrm{NO}_{2}$ & + & 3.28 & 234.14885 & 125.0 & 3.62 & 92.7 & 12.50 & 1.12 \\
\hline Mitragyne & $\mathrm{C}_{23} \mathrm{H}_{30} \mathrm{~N}_{2} \mathrm{O}_{4}$ & + & 4.75 & 399.22783 & 125.0 & 6.59 & 91.7 & 12.50 & 0.41 \\
\hline Modafinil & $\mathrm{C}_{15} \mathrm{H}_{15} \mathrm{NO}_{2} \mathrm{~S}$ & + & 4.85 & 296.07157 & 125.0 & 4.87 & 92.9 & 12.50 & 0.14 \\
\hline Nikethamine & $\mathrm{C}_{10} \mathrm{H}_{14} \mathrm{~N}_{2} \mathrm{O}$ & + & 2.84 & 179.11789 & 62.5 & 15.14 & 8.3 & 6.25 & 0.00 \\
\hline Norfenfluramine & $\mathrm{C}_{10} \mathrm{H}_{12} \mathrm{~F}_{3} \mathrm{~N}$ & + & 3.60 & 204.09946 & 125.0 & 3.05 & 91.5 & 12.50 & 1.47 \\
\hline Octhylamine & $\mathrm{C}_{8} \mathrm{H}_{18} \mathrm{~N}$ & + & 4.35 & 130.15903 & 125.0 & 2.91 & 88.8 & 12.50 & 0.98 \\
\hline Oxilofrine & $\mathrm{C}_{10} \mathrm{H}_{15} \mathrm{NO}_{2}$ & + & 0.39 & 133.06479 & 125.0 & 7.63 & 94.8 & 12.50 & 3.80 \\
\hline
\end{tabular}


Determination of adulterants in whey protein food supplements by liquid chromatography coupled to Orbitrap high resolution mass spectrometry

Roiffé, R. R. et al.

Table 1. Continued...

\begin{tabular}{|c|c|c|c|c|c|c|c|c|c|}
\hline \multirow[b]{2}{*}{ Compound } & \multirow[b]{2}{*}{ Chemical Formula } & \multirow[b]{2}{*}{ Polarity } & \multirow[b]{2}{*}{$t_{R}(\min )$} & \multirow[b]{2}{*}{$m / z$} & \multirow{2}{*}{$\frac{\mathrm{SC}}{\left(\mathrm{ng} \mathrm{g}^{-1}\right)}$} & \multirow{2}{*}{$\begin{array}{c}\text { Repeatability } \\
(\%)\end{array}$} & \multirow{2}{*}{$\begin{array}{c}\text { Extraction } \\
\begin{array}{c}\text { yield } \\
(\%)\end{array}\end{array}$} & \multirow{2}{*}{$\frac{\text { LOD }}{(\text { ng.g }}$} & \multirow{2}{*}{$\begin{array}{c}\text { Matrix } \\
\text { interference } \\
(\%) \\
\end{array}$} \\
\hline & & & & & & & & & \\
\hline Pemoline & $\mathrm{C}_{9} \mathrm{H}_{8} \mathrm{~N}_{2} \mathrm{O}$ & + & 2.09 & 177.06585 & 125.0 & 32.90 & 4.7 & 12.50 & 0.25 \\
\hline Pentetrazol & $\mathrm{C}_{6} \mathrm{H}_{10} \mathrm{~N}_{4}$ & + & 2.03 & 139.09782 & 125.0 & 23.38 & 6.2 & 12.50 & 0.25 \\
\hline Phendimetrazine & $\mathrm{C}_{12} \mathrm{H}_{17} \mathrm{NO}$ & + & 1.88 & 192.13829 & 125.0 & 2.68 & 91.3 & 12.50 & 3.65 \\
\hline Phenmetrazinha & $\mathrm{C}_{11} \mathrm{H}_{16} \mathrm{CINO}$ & + & 1.92 & 178.12264 & 125.0 & 3.20 & 91.4 & 12.50 & 1.73 \\
\hline Pholedrine & $\mathrm{C}_{10} \mathrm{H}_{15} \mathrm{NO}$ & + & 0.89 & 166.12264 & 125.0 & 9.77 & 94.6 & 12.50 & 4.30 \\
\hline p-hydroxy amphetamine & $\mathrm{C}_{9} \mathrm{H}_{13} \mathrm{NO}$ & + & 0.85 & 135.08044 & 125.0 & 8.73 & 99.4 & 12.50 & 4.30 \\
\hline Pipradol & $\mathrm{C}_{18} \mathrm{H}_{21} \mathrm{NO}$ & + & 4.07 & 268.16959 & 125.0 & 3.06 & 93.5 & 12.50 & 1.08 \\
\hline Prenilamyne & $\mathrm{C}_{24} \mathrm{H}_{27} \mathrm{~N}$ & + & 6.52 & 330.22163 & 125.0 & 11.48 & 90.2 & 12.50 & 0.12 \\
\hline Prolintane & $\mathrm{C}_{15} \mathrm{H}_{23} \mathrm{~N}$ & + & 3.82 & 218.19033 & 125.0 & 2.18 & 91.5 & 12.50 & 0.98 \\
\hline Propylhexedrine & $\mathrm{C}_{10} \mathrm{H}_{21} \mathrm{~N}$ & + & 3.79 & 156.17468 & 125.0 & 2.69 & 93.0 & 12.50 & 1.21 \\
\hline $\mathrm{s}(+)$-methamphetamine & $\mathrm{C}_{10} \mathrm{H}_{15} \mathrm{~N}$ & + & 2.05 & 150.12773 & 62.5 & 3.90 & 91.5 & 6.25 & 1.42 \\
\hline Selegine & $\mathrm{C}_{13} \mathrm{H}_{17} \mathrm{~N}$ & + & 2.54 & 188.14338 & 125.0 & 5.28 & 88.2 & 12.50 & 1.24 \\
\hline Sibutramine & $\mathrm{C}_{17} \mathrm{H}_{26} \mathrm{CIN}$ & + & 6.06 & 280.18265 & 125.0 & 7.17 & 86.4 & 12.50 & 0.13 \\
\hline Strychnine & $\mathrm{C}_{21} \mathrm{H}_{22} \mathrm{~N}_{2} \mathrm{O}_{2}$ & + & 2.53 & 335.17540 & 125.0 & 3.97 & 90.4 & 12.50 & 1.29 \\
\hline Trimetazidine & $\mathrm{C}_{14} \mathrm{H}_{22} \mathrm{~N}_{2} \mathrm{O}_{3}$ & + & 1.86 & 267.17032 & 125.0 & 7.17 & 82.2 & 12.50 & 1.20 \\
\hline
\end{tabular}


Table 1 summarizes the results observed for all the substances validated. The detection limit (LOD) represents the lowest concentration of the substance that is detectable but not necessarily quantified using an experimental procedure. The LOD for anabolic agents was $1.25 \mathrm{ng} \mathrm{g}^{-1}$, beta-agonists from 5 to $10 \mathrm{ng} \mathrm{g}^{-1}$, hormone and metabolic modulators from 5 to $12.5 \mathrm{ng} \mathrm{g}^{-1}$, diuretics from 6.25 to $62.5 \mathrm{ng} \mathrm{g}^{-1}$, and stimulants and anorectic agents from 3.16 to $25 \mathrm{ng} \mathrm{g}^{-1}$. All compounds were detected with more than 8 point-acquisitions at the LOD. This low limit of detection is associated with the screening method sensitivity and it is important for the screening of adulterants in commercial WPFS samples.

The repeatability was verified using the peak areas of each analyte from 7 WPFS replicates and Table 1 shows this variation as the relative standard deviation (RSD). The RSD values obtained were compared with the maximum relative standard deviation for each level of concentration calculated by the Horwitz equation (Horwitz et al., 1980). According to the comparison between the RSD and the values obtained using the Horwitz equation, the majority of the substances monitored (96.19\%) showed an adequate relative standard deviation, indicating a low result dispersion. Only ethacrynic acid, benzbromarone, bumetanide and fulvestrant showed RSD values above the values preconized by the Horwitz equation.

The recovery can also be observed in Table 1 and it was lower than $50 \%$ for $15 \%$ of the targeted substances including ethacrynic acid, benzbromarone, bumetanide and fulvestrant again, and also clopamide, chlorothiazide, chlorthalidone, diclofenamide, hydrochlorothiazide, hydroflumethiazide, methazolamide, piretanide, probenecid, carphedon, methylenedioxyamfetamine, nikethamide, pemoline, pentetrazol, trichlormethiazide and xipamide. Almost all of these substances are acids. However, despite the recovery below $50 \%$, all the substances were properly detected in all the replicates, mainly because by HRMS, low noise and clear signals were obtained, allowing the presence of the substances to be detected.

As a consequence of this comprehensive sample preparation procedure, a high influence of the matrix in the instrumental conditions was expected. When complex matrices such as WPFS are analyzed, signal suppression of an analyte can occur and/or a shift in the retention time can be observed, probably because of the punctual modification of the stationary phase or due to overlay of the WPFS matrix components. The ion suppression was evaluated in the matrix effect experiments and the variation in the retention time $\left(t_{R}\right)$ was evaluated by monitoring the $t_{\mathrm{R}}$ peak for all targeted substances for their respective retention times in a window of 1 minute. After injecting 10 different replicates, the highest RSD observed was $2 \%$ for the $t_{R}$ of the substances that elute before 1 minute (Table 1).

Finally, the existence of carryover was tested, but none was observed. Carryover verifies the existence of significant variations amongst sequential injections.

\subsection{Application to the commercial samples}

The eleven brands of WPFS (identified by the numbers 1 to 11) were analyzed and compared with the positive control to check for the presence of adulterants. According to the LC-Orbitrap-HRMS analysis, peaks with retention times and $\mathrm{m} / \mathrm{z}$ ratios equal or similar (error below $5 \mathrm{ppm}$ ) to the substances conivaptan, polythiazide and benfluorex, were found in some commercial samples. The samples showing a suspicion of adulteration were extracted and analysed twice more to confirm the presence of the adulterants.

Suspicious samples were confirmed based mainly on the parameters of the mass/charge ratios of the precursor ions, mass accuracy calculation, the RDBE values of the samples, and comparison with the positive control. Variations in the $t_{R}$ and $m / z$ ratios of the positive control and the commercial samples were observed in the suspect peaks. Subsequently the mass accuracy was calculated (maximum limit of $5 \mathrm{ppm}$ for confirmation of the identities of the compounds) and the RDBE values. Figure 1 shows the retention times $\left(t_{R}, \min \right)$, molecular ions, calculation of mass accuracy, and the RDBE values relevant to the presence of the following diuretics: conivaptan, polythiazide and/or the stimulant: benfluorex. 

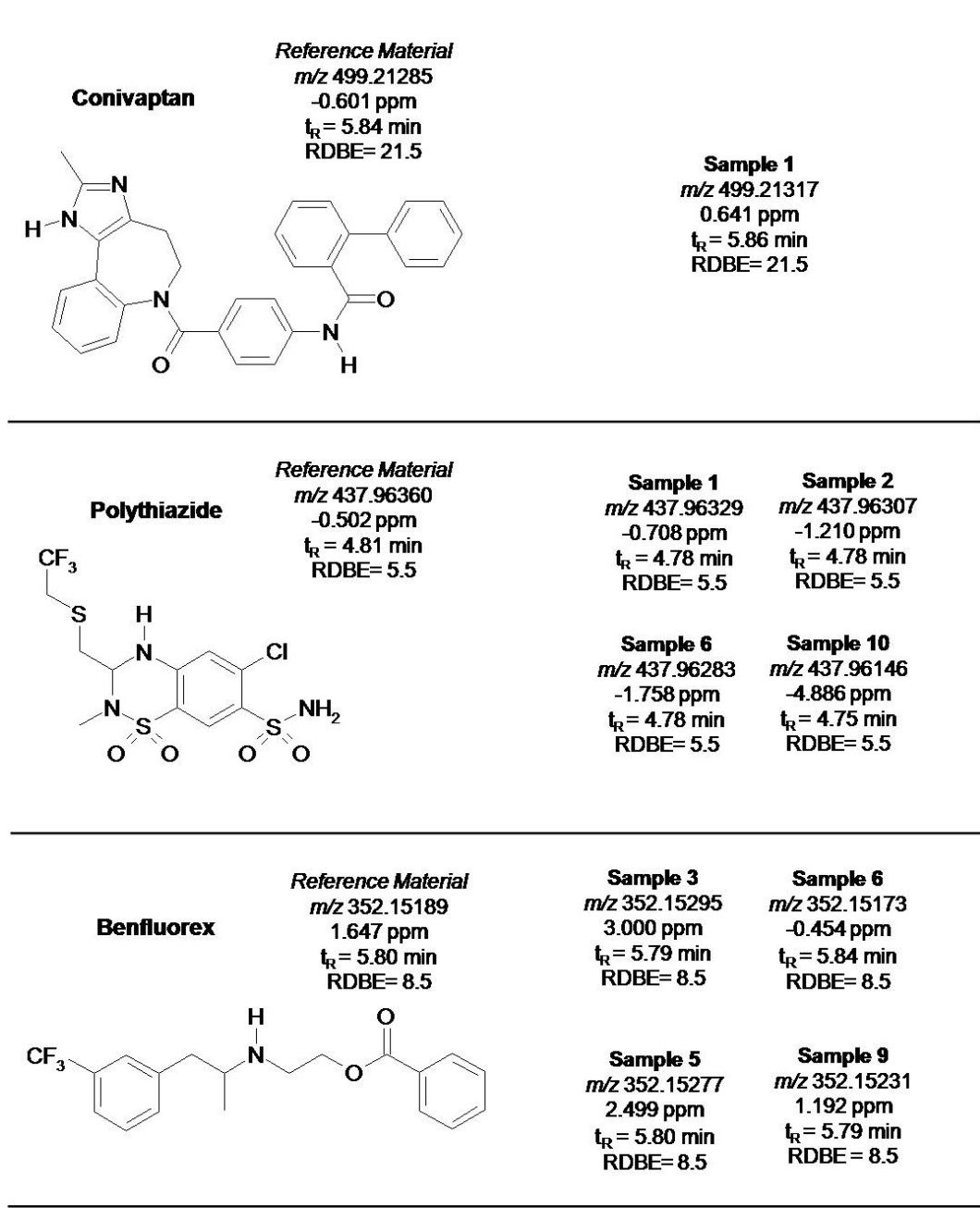

Figure 1. Chemical structures, $m / z$, ppm error, retention time and RDBE of conivaptan, polythiazide and benfluorex in the reference material and in the samples.

According to the comparison of the parameters, the values obtained for the samples suspected of adulteration were compatible with those of the positive control, confirming the presence of adulterants in those whey protein food supplements. After applying the method, seven of the eleven brands analysed (63.64\%) showed adulteration by at least one of the above-mentioned substances.

The administration of these compounds can cause health risks (depending on the associated factors) and another aggravating fact is that many individuals are consuming products classified as foods without knowing that they may contain substances with pharmacological properties. The adulteration by diuretics and/or anorectic stimulants is related to the effects they may cause, and an effective weight loss is amongst the effects common to these classes. Conivaptan and polythiazide act by increasing diuresis, masking the other substances present, and benfluorex is a stimulant with an anorectic effect that induces a loss of appetite (Docherty, 2008; Woo et al., 2013).

\section{Conclusions}

Whey protein food supplement samples contain a high concentration of proteins which can be removed by solvent precipitation and solid-phase extraction clean-up before sample injection into the chromatographic system. Cold water was the best solvent option to remove these proteins and maintain the procedure comprehensive. 
The LC-Orbitrap-HRMS method allowed for better separation, detection and identification of the analytes, due to the high sensitivity and resolution of the mass analyser.

The parameters selectivity, LOD, repeatability, extraction yield, carryover and matrix effect were duly validated. All these parameters showed satisfactory results in the detection of the substances with pharmacological action in the WPFS matrix.

After applying the method, it was shown that four commercial samples were not adulterated and seven were. One showed the presence of both conivaptan and politiazide (a combination of diuretic agents), two showed the presence of politiazide and benfluorex, and the others only showed the presence of either politiazide or benfluorex.

\section{Acknowledgements}

This work was supported by the Coordenação de Aperfeiçoamento de Pessoal de Nivel Superior (CAPES), Laboratório Brasileiro de Controle de Dopagem (LBCD) and by the Instituto Militar de Engenharia (IME).

\section{References}

Andrade, J., Pereira, C. G., Almeida Junior, J. C., Viana, C. C. R., Neves, L. N. O., Silva, P. H. F., Bella, M. J. V., \& Anjos, V. C. (2019). FTIR-ATR determination of protein content to evaluate whey protein concentrate adulteration. Food Science and Technology, 99, 166-172.

Aquino, L. F. M. C., Ribeiro, R. O. R., Simoes, J. S., Mano, S. B., Mársico, E. T., \& Conte Junior, C. A. (2017). Mercury content in whey protein and potential risk for human health. Journal of Food Composition and Analysis, 59, 141-144. http://dx.doi.org/10.1016/j.jfca.2017.02.014

Associação Brasileira de Normas Técnicas - ABNT. (2005). NBR ISO/IEC 17025: General requirements for the competence of testing and calibration laboratories. Rio de Janeiro: ABNT.

Bagchi, D., Nair, S., \& Sen, C. K. (2013). Nutrition and enhanced sports performance: Muscle building, endurance, and strength (1st ed). United States: Academic Press.

Chen, W., Huang, W., Chiu, C., Chang, W., \& Huang, C. (2014). Whey protein improves exercise performance and biochemical profiles in trained mice. Medicine and Science in Sports and Exercise, 46(8), 1517-1524. PMid:24504433. http://dx.doi.org/10.1249/MSS.0000000000000272

Docherty, J. R. (2008). Pharmacology of stimulants prohibited by the World Anti-Doping Agency (WADA). British Journal of Pharmacology, 154(3), 606-622. PMid:18500382. http://dx.doi.org/10.1038/bjp.2008.124

Fayh, A. P. T., Silva, C. V., Jesus, F. R. D., \& Costa, G. K. (2013). Consumo de suplementos nutricionais por freqüentadores de academias da cidade de Porto Alegre. Revista Brasileira de Ciências do Esporte, 35(1), 27-37. http://dx.doi.org/10.1590/S010132892013000100004

Frestedt, J. L., Zenk, J. L., Kuskowski, M. A., Ward, L. S., \& Bastian, E. D. (2008). A whey-protein supplement increases fat loss and spares lean muscle in obese subjects: A randomized human clinical study. Nutrition \& Metabolism, 5(8), 1-7. PMid:18371214. http://dx.doi.org/10.1186/1743-7075-5-8

Garrido, B. C., Souza, G. H. M. F., Lourenço, D. C., \& Fasciotti, M. (2016). Proteomics in quality control: Whey protein-based supplements. Journal of Proteomics, 147, 48-55. PMid:27072112. http://dx.doi.org/10.1016/j.jprot.2016.03.044

Haraguchi, F. K., Abreu, W. C., \& Paula, H. (2006). Proteínas do soro do leite: Composição, propriedades nutricionais, aplicações no esporte e benefícios para a saúde humana. Revista de Nutrição, 19(4), 479-488. http://dx.doi.org/10.1590/S141552732006000400007

Hernandez, A. J., \& Nahas, R. M. (2009). Modificações dietéticas, reposição hídrica, suplementos alimentares e drogas: Comprovação de ação ergogênica e potenciais riscos para a saúde. Revista Brasileira de Medicina do Esporte, 15(3), 3-12.

Horikawa, Y., Tsutsumi, R., \& Tsutsumi, Y. (2013). Whey protein diets can limit inflammation and oxidative stress in the critically ill. Critical Care Medicine, 41(12), A181-A182. http://dx.doi.org/10.1097/01.ccm.0000439972.21054.05

Horwitz, W., Kamps, L. R., \& Boyer, R. W. (1980). Quality assurance in the analysis of foods and trace constituents. Journal Association of Official Analytical Chemists, 63(6), 1344-1354. PMid:7451398.

Lu, Y. L., Zhou, N. L., Liao, S. Y., Su, N., He, D. X., Tian, Q. Q., Chen, B., \& Yao, S. Z. (2010). Detection of adulteration of antihypertension dietary supplements and traditional Chinese medicines with synthetic drugs using LC/MS. Food Additive and Contaminants: Part A, 27(7), 893-902. PMid:20544454. http://dx.doi.org/10.1080/19440040903426710

Marcus, D. M. (2016). Dietary supplements: What's in a name? What's in the bottle? Drug Testing and Analysis, 8(3-4), 410412. PMid:27072845. http://dx.doi.org/10.1002/dta.1855 
Martello, S., Felli, M., \& Chiarotti, M. (2007). Survey of nutritional supplements for selected illegal anabolic steroids and ephedrine using LC-MS/MS and GC-MS methods, respectively. Food Additives and Contaminants, 24(3), 258-265. PMid:17364927. http://dx.doi.org/10.1080/02652030601013729

Martínez-Sanz, J. M., Sospedra, I., Ortiz, C. M., Baladía, E., Gil-Izquierdo, A., \& Ortiz-Moncada, R. (2017). Intended or unintended doping? A review of the presence of doping substances in dietary supplements used in sports. Nutrients, $9(10)$, 1093. PMid:28976928. http://dx.doi.org/10.3390/nu9101093

Mathews, N. M. (2018). Prohibited contaminants in dietary supplements. Sports Health, 10(1), 19-30. PMid:28850291. http://dx.doi.org/10.1177/1941738117727736

Maughan, R. J., Burke, L. M., Dvorak, J., Larson-Meyer, D. E., Peeling, P., Phillips, S. M., Rawson, E. S., Walsh, N. P., Garthe, I., Geyer, H., Meeusen, R., van Loon, L., Shirreffs, S. M., Spriet, L. L., Stuart, M., Vernec, A., Currell, K., Ali, V. M., Budgett, R. G. M., Ljungqvist, A., Mountjoy, M., Pitsiladis, Y., Soligard, T., Erdener, U., \& Engebretsen, L. (2018). IOC consensus statement: Dietary supplements and the high-performance athlete. International Journal of Sport Nutrition and Exercise Metabolism, 28(2), 104-125. PMid:29589768. http://dx.doi.org/10.1123/ijsnem.2018-0020

McClung, J. P., \& Murray-Kolb, L. E. (2013). Iron nutrition and premenopausal women: Effects of poor iron status on physical and neuropsychological performance. Annual Review of Nutrition, 33(1), 271-288. PMid:23642204. http://dx.doi.org/10.1146/annurev-nutr-071812-161205

Müller, L. S., Muratt, D. T., Molin, T. R. D., Urquhart, C. G., Viana, C., \& Carvalho, L. M. (2018). Analysis of pharmacologic adulteration in dietary supplements by capillary zone electrophoresis using simultaneous contactless conductivity and UV detection. Chromatographia, 81(4), 689-698. http://dx.doi.org/10.1007/s10337-018-3496-2

Neves, D. B. J., \& Caldas, E. D. (2015). Dietary supplements: International legal framework and adulteration profiles, and characteristics of products on the Brazilian clandestine market. Regulatory Toxicology and Pharmacology, 73(1), 93-104. PMid:26107294. http://dx.doi.org/10.1016/j.yrtph.2015.06.013

Parra, R. M. T., Palma, A., \& Pierucci, A. P. T. R. (2011). Contaminação de suplementos dietéticos usados para prática esportiva. Revista Brasileira de Ciências do Esporte, 33(4), 1071-1084.

Pritchard-Peschek, K. R., Osborne, M. A., Slater, G. J., Taaffe, D. R., \& Jenkins, D. G. (2013). Pseudoephedrine and preexercise feeding: Influence on performance. Medicine and Science in Sports and Exercise, 45(6), 1152-1157. PMid:23274597. http://dx.doi.org/10.1249/MSS.0b013e3182808e23

Roco, M. C., \& Bainbridge, W. S. (2002). Converging technologies for improving human performance. Journal of Nanoparticle Research, 4(4), 281-295. http://dx.doi.org/10.1023/A:1021152023349

Rondanelli, M., Klersy, C., Terracol, G., Talluri, J., Maugeri, R., Guido, D., Faliva, M. A., Solerte, B. S., Fioravanti, M., Lukaski, H., \& Perna, S. (2016). Whey protein, amino acids, and vitamin D supplementation with physical activity increases fat-free mass and strength, functionality, and quality of life and decreases inflammation in sarcopenic elderly. The American Journal of Clinical Nutrition, 103(3), 830-840. PMid:26864356. http://dx.doi.org/10.3945/ajcn.115.113357

Sardela, V. F., Martucci, M. E. P., de Araújo, A. L. D., Leal, E. C., Oliveira, D. S., Carneiro, G. R. A., Deventer, K., Van Eenoo, P., Pereira, H. M. G., \& Aquino Neto, F. R. (2018). Comprehensive analysis by liquid chromatography Q-Orbitrap mass spectrometry: Fast screening of peptides and organic molecules. Journal of Mass Spectrometry, 53(6), 476-503. PMid:29524299. http://dx.doi.org/10.1002/jms.4077

Thomas, J. R., Silverman, S., \& Nelson, J. (2015). Research methods in physical activity (7th ed.). United States: Human Kinetics.

Woo, H., Kim, J. W., Han, K. M., Lee, J. H., Hwang, I. S., Lee, J. H., Kim, J., Kweon, S. J., Cho, S., Chae, K. R., Han, S. Y., \& $\mathrm{Kim}, \mathrm{J}$. (2013). Simultaneous analysis of 17 diuretics in dietary supplements by HPLC and LC-MS/MS. Food Additive and Contaminants: Part A, 30(2), 209-217. PMid:23116261. http://dx.doi.org/10.1080/19440049.2012.738939 